\title{
Investigations on Structural, Mechanical, and Dielectric Properties of PVDF/Ceramic Composites
}

\author{
Anshuman Srivastava, ${ }^{1}$ Karun Kumar Jana, ${ }^{2}$ Pralay Maiti, ${ }^{2}$ \\ Devendra Kumar, ${ }^{1}$ and Om Parkash ${ }^{1}$ \\ ${ }^{1}$ Department of Ceramic Engineering, Indian Institute of Technology (Banaras Hindu University), Varanasi 221005, India \\ ${ }^{2}$ School of Material Science and Technology, Indian Institute of Technology (Banaras Hindu University), Varanasi 221005, India \\ Correspondence should be addressed to Om Parkash; oprakash.cer@itbhu.ac.in
}

Received 29 August 2014; Revised 17 December 2014; Accepted 18 December 2014

Academic Editor: H. P. S. Abdul Khalil

Copyright ( 2015 Anshuman Srivastava et al. This is an open access article distributed under the Creative Commons Attribution License, which permits unrestricted use, distribution, and reproduction in any medium, provided the original work is properly cited.

Polymer ceramic composites are widely used for embedded capacitor application. In the present work PVDF has been used as a matrix and CCTO and LaCCTO have been used as reinforcement. Extrusion process has been used for the synthesis of composites. $\mathrm{X}$-ray diffraction (XRD) patterns confirm the formation of single phase CCTO, and LaCCTO in its pure as well as composite state. It is found that La doping in CCTO considerably increases the dielectric constant and reduces the dielectric loss. A similar trend is observed in the composites with the increasing content of CCTO and LaCCTO.

\section{Introduction}

Recently, polymer ceramic composites have attracted a lot of interest in industrial applications because of dramatic improvement that can be made in their properties by varying the type and amount of dispersion. Modern electronic devices demand new high dielectric constant materials with low dielectric loss and enhanced dielectric strength $[1-3]$. These materials should fulfill the industrial need of suitable dielectric properties, improved mechanical strength, and ease of processing at a relative low cost. Polymer ceramic composites can be used in various applications including integrated capacitors, acoustic emission sensors, smart skins, and leakage current controllers [1-4].

The possibility of developing composites by incorporating ferroelectric ceramics in polymer matrix has been investigated by many workers. Incorporation of ferroelectric ceramic (such as barium titanate, $\mathrm{Pb}\left(\mathrm{Mg}_{1 / 3} \mathrm{Nb}_{2 / 3}\right) \mathrm{O}_{3} \mathrm{PbTiO}_{3}$, and PMN-PT) has been studied by many researchers [512]. Ferroelectric materials undergo phase transition at a characteristic temperature, known as Curie temperature. Piezoelectric materials and polar oxides exhibit low structural symmetry. Another drawback with these composites is that even at very high content of reinforcement dielectric constant does not become more than 50 [13-17].
As compared to ferroelectric materials, CCTO has advantage from the application point of view in electronic devices such as capacitors, dynamic random access memories, varistors, and thermistors. $\mathrm{CaCu}_{3} \mathrm{Ti}_{4} \mathrm{O}_{12}$ has a distorted and complex cubic perovskite-like structure with large unit cell $(\sim 7.4 \AA)$ and its dielectric constant remains unchanged over a wide range of frequencies and temperatures [18-20]. So many theories have been proposed to explain the abnormal dielectric properties of CCTO. Among the various explanations, the internal barrier layer capacitance mechanism has been widely accepted [21]. According to the IBLC model, CCTO ceramic can be considered as a composite material consisting of the semiconducting grains and the insulating grain boundaries. Grains possess small resistivity and the insulating grain boundaries possess giant resistivity. Grain boundary inhibits the flow of electrons in grain and causes a large boundary polarization which results in the giant dielectric constant.

Poly(vinylidene fluoride) (PVDF) is a chemically, thermally, and mechanically very stable material. It has excellent ferroelectric, pyroelectric, and piezoelectric properties [2224]. Dielectric constant of more than 610 at $10^{2} \mathrm{~Hz}$ at room temperature in $\mathrm{CCTO} / \mathrm{P}(\mathrm{VDF}-\mathrm{TrFE})$ when the filler volume is 50\% was reported by Arbatti et al. [22]. Yang et al. studied 
the effect of coupling agent on PVDF-CCTO composites using $0.1 \mathrm{~mL}$ of Si69 coupling agent. A value of $\varepsilon^{\prime}, 84$, for PVDF/CCTO composite was obtained, and $\varepsilon^{\prime}$ value was 16 in case of PVDF/CCTO composite without coupling agent [23]. Zhang et al. reported a dielectric constant of 62 having a loss tangent of 0.05 for nanocomposites containing $50 \mathrm{vol} \%$ CCTO at room temperature and $1 \mathrm{KHz}$ [24]. Ramajo et al. studied the dielectric behavior of CCTO-epoxy composites containing up to 15 volume \% of CCTO [25]. Thomas et al. found a room temperature value 95 of dielectric constant at $100 \mathrm{~Hz}$ for 55 volume \% of CCTO in PVDF [26]. Yang et al. studied the particle size effect on the dielectric properties of CCTO/PVDF composites [27]. For nanosize CCTO at $40 \mathrm{vol} \%$, they got a value of $\varepsilon^{\prime}>10^{6}$ at $10^{2} \mathrm{~Hz}$ at room temperature which was much higher than the value of 35.7 for microsize CCTO at the same content. Effect of interfacial polarization was found to be a reason for high value of $\varepsilon^{\prime}$ for nanosize powder. Interface between filler and matrix was proposed to explain the properties of composites containing microsize CCTO particles.

Dang et al. reported a dielectric constant $\sim 49$ at $100 \mathrm{~Hz}$ and room temperature for composite film of CCTO/polyimide [28]. Prakash and Verma reported a value of 8.3 and 50 for $\varepsilon^{\prime}$ at $10 \mathrm{KHz}$ and $300 \mathrm{~K}$ for 10 volume $\%$ and 30 volume $\%$, respectively, in the epoxy matrix [29]. Dielectric and mechanical properties of PVDF-La modified CCTO (LaCCTO) containing $10 \mathrm{wt} \%$ of LaCCTO have been reported earlier by us. It was observed that dielectric constant of PVDF increased from 5 to 30 in case of PVDF-LaCCTO composite $(10 \mathrm{wt} \%)$ at $1 \mathrm{~Hz}$ and $40^{\circ} \mathrm{C}$ [30]. Dielectric constant 98 has been achieved for $50 \mathrm{wt} \% \mathrm{NbCCTO/PVDF}$ composite at $40^{\circ} \mathrm{C}$ and $100 \mathrm{~Hz}$ [31].

Most of the researchers remained confined to PVDFCCTO composites. They focused their study on the dielectric behaviour as a function of CCTO content.

All these studies were confined to dielectric properties. Most of the investigations done so far have reported the dielectric behaviour of the composites containing a very high content of the ceramic fillers. High content of the ceramic in polymer results in the deterioration of mechanical properties due to agglomeration and porosity. In the present investigations efforts have been made to develop high dielectric constant composites at low filler content. In this paper mechanical properties have also been reported along with dielectric properties. Mechanical behaviour is as important as the dielectric properties for applications in the devices.

\section{Experimental}

2.1. Materials. Poly(vinylidene fluoride) (PVDF) (SOLEF 6008; Ausimont, Italy) with a melt flow index of $24 \mathrm{~g} / 10 \mathrm{~min}$ at $230^{\circ} \mathrm{C}$ under $5 \mathrm{~kg}$ load was used as polymer matrix. To synthesise samples of $\mathrm{CaCu}_{3} \mathrm{Ti}_{4} \mathrm{O}_{12}$ and $\mathrm{Ca}_{(1-3 x / 2)} \mathrm{La}_{x} \mathrm{Cu}_{3} \mathrm{Ti}_{4} \mathrm{O}_{12}$ $(x=0.05)$ semiwet route was used. Analytical grade chemicals, $\mathrm{Ca}\left(\mathrm{NO}_{3}\right)_{2} \cdot 4 \mathrm{H}_{2} \mathrm{O}, \mathrm{La}\left(\mathrm{NO}_{3}\right)_{3} \cdot 6 \mathrm{H}_{2} \mathrm{O}, \mathrm{Cu}\left(\mathrm{NO}_{3}\right)_{2} \cdot 3 \mathrm{H}_{2} \mathrm{O}$, titanium dioxide, and citric acid, having purity better than 99.95\% were used as starting materials. Solutions of nitrates of these elements in the stoichiometric amount were mixed in a beaker. Calculated amount of $\mathrm{TiO}_{2}$ and citric acid equivalent

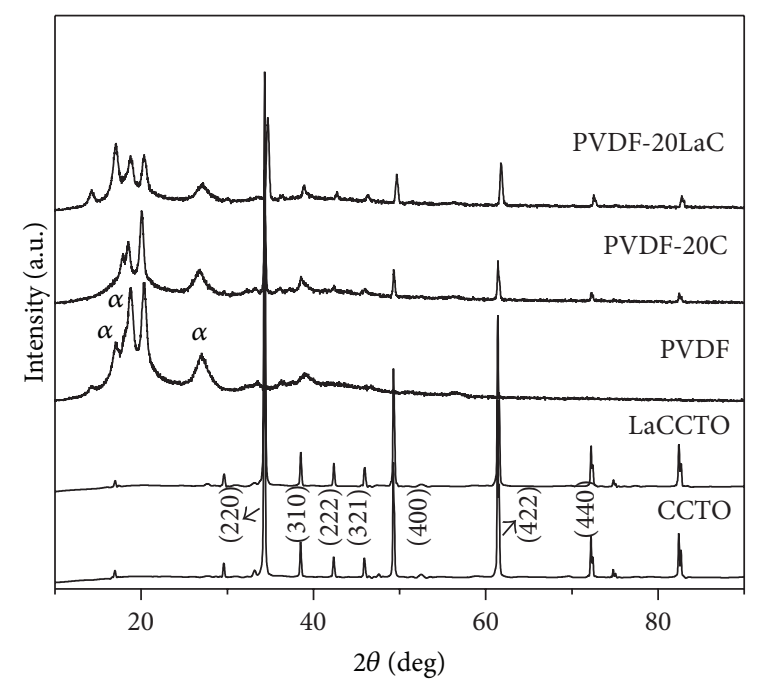

FIGURE 1: X-ray diffraction patterns for CCTO, pure PVDF, PVDF20C, and PVDF-20LaC composites.

to metal ions was added to the mixed solution. The mixture was heated at $80-90^{\circ} \mathrm{C}$ on a hot plate to evaporate water and then dried at $110-120^{\circ} \mathrm{C}$ in hot air oven for $12 \mathrm{~h}$ to yield a blue dry powder. Differential thermal analysis (DTA) and thermogravimetric analysis (TGA) of the powder were carried out from 30 to $1000^{\circ} \mathrm{C}$ at a heating rate of $10^{\circ} \mathrm{C} / \mathrm{min}$ in air using Perkin-Elmer, USA, TGA/DTA Analyzer. Calcination was done at $800^{\circ} \mathrm{C}$ in an electrical furnace for $7 \mathrm{~h}$. After calcination, pellets of the calcined powder were made using a hydraulic press and sintered at $900^{\circ} \mathrm{C}$ for $6 \mathrm{~h}$. X-ray diffraction (XRD) patterns of the sintered pellets confirmed the formation of single phase solid solution.

2.2. Composite Preparation. Extrusion process was used to prepare PVDF/CCTO (PVDF-20C) and PVDF/LaCCTO (PVDF-20LaC) composites. Before extrusion, 12 gm of polymer was mixed with $20 \mathrm{wt} \%$ of ceramics in a high speed mixer for $30 \mathrm{~min}$. Extrusion was carried out in a twin-screw extruder (Hakke Mini Lab). Mixing was done at $210^{\circ} \mathrm{C}$ for $\sim 15$ minutes under high shear rate at $70 \mathrm{rpm}$. During melt mixing, ceramic particles mix uniformly in polymer chains. PVDF and composites were melt-pressed into a thin film of $100 \mu \mathrm{m}$ thickness in a compression-molding machine at $200^{\circ} \mathrm{C}$ under 6 tons of pressure for experimental purpose.

2.3. Characterization. X-ray diffraction (XRD) patterns were recorded using a Rigaku Desktop Miniflex II X-Ray diffractometer employing $\mathrm{Cu}-\mathrm{K} \alpha$ radiation and Ni-filter (wavelength, $\lambda=0.154 \mathrm{~nm}$ ). Thin films of PVDF and composites were scanned in the diffraction angle $2 \theta$ range from 10 to $90^{\circ}$ at a scan rate of $3^{\circ} / \mathrm{min}$. SEM images were recorded using INSPECT S 50 FP 2017/12 Scanning Electron Microscope. Gold coating was done on the surface of samples to make them conducting.

Instron 3369 tensile machine was used for tensile test of dog bone shaped samples at room temperature. A constant 

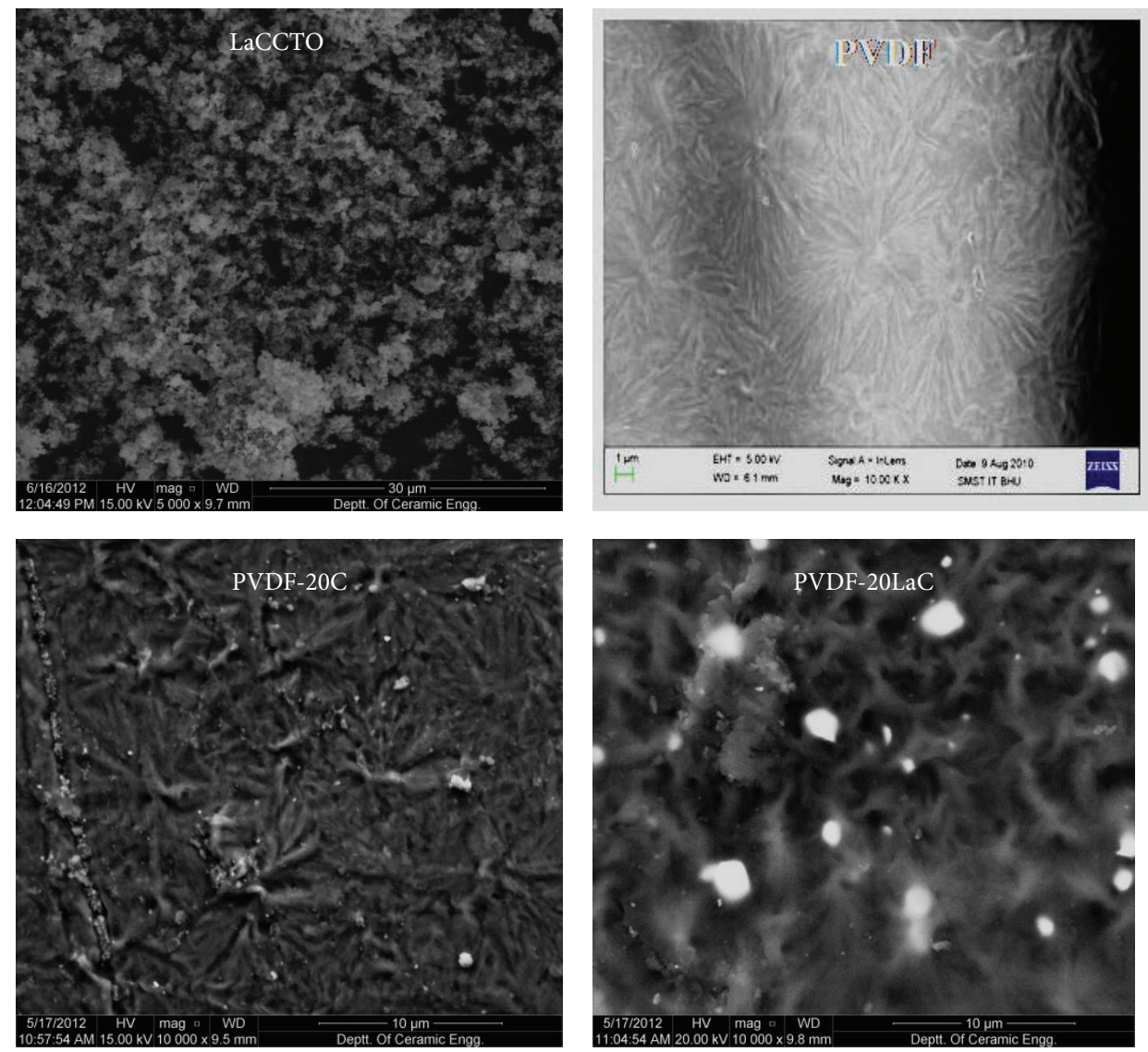

Figure 2: Scanning electron micrographs of LaCCTO, PVDF, PVDF-20C, and PVDF-20LaC composites.

crosshead speed of $5 \mathrm{~mm} / \mathrm{min}$ was selected and the stressstrain data were recorded up to the complete breaking of most samples. Three samples were tested for each specimen.

Contact angle measurements of pure PVDF and composites were done using Kruss (Processor) Tensiometer K 100. The strips were immersed in water at room temperature and the contact angle was measured in the advance mode.

Dielectric measurements were performed on silver coated disc-shaped films of $14 \mathrm{~mm}$ diameter. Measurements were carried out in the frequency range $10^{-2}-10^{6} \mathrm{~Hz}$ using fourprobe Novocontrol setup (ZG4) in the temperature range of room temperature to $80^{\circ} \mathrm{C}$.

\section{Results and Discussion}

3.1. Structural Analysis. X-ray diffraction patterns of CCTO, LaCCTO, pure PVDF, and composites are shown in Figure 1. Diffraction peaks corresponding to the planes (220), (310), (222), (321), (400), (422), and (440) confirmed the formation of single phase compound of CCTO and LaCCTO. No evidence of any secondary phase was found in LaCCTO. Pure PVDF crystallizes in $\alpha$ phase with characteristic peaks at $17.7^{\circ}$, $18.7^{\circ}$, and $19.9^{\circ}$, corresponding to (110), (020), and (111) crystal planes, respectively [26]. Diffraction peaks corresponding to (220), (400), and (422) planes in PVDF-20C and PVDF$20 \mathrm{LaC}$ show the presence of CCTO and LaCCTO and successful formation of composites.
3.2. Surface Morphology. Figure 2 shows the SEM micrographs of pure PVDF, LaCCTO, and composites. PVDF exists in spherulitic morphology which is getting severely affected by ceramic dispersion in composites. This indicates homogeneous distribution of ceramic particles in PVDF matrix. It has been reported earlier that good dispersion along with homogeneous packing of ceramic filler is likely to exhibit high dielectric constant [26]. Contact angle measurements of pure PVDF and composite samples were done using Kruss (Processor) Tensiometer K 100. For PVDF it was $71^{\circ}$, and for PVDF-20C and PVDF-20LaC composites it was found to be $74^{\circ}$ and 77 , respectively. This shows that composites are slightly more hydrophobic than PVDF, that is, more chemical resistant to aqueous medium.

3.3. Mechanical Properties. Dog bone shaped samples were made by using injection molding technique (microinjector, model FD-1, Fly Tech Engineering). Temperature of the mould was kept at $70^{\circ} \mathrm{C}$ and that of the cylinder at $215^{\circ} \mathrm{C}$ under a pressure of 100 bars. Sample prepared by this method had a cross-sectional dimension of $(2.15 \times 4) \mathrm{mm}^{2}$, and the length of the gauge section was kept $20 \mathrm{~mm}$. Stress-strain curves of PVDF and composites are shown in Figures 3(a)3(c). Young's modulus increases considerably. For PVDF modulus value is $920 \mathrm{MPa}$. In case of composites, modulus value increased to 1154 and $1363 \mathrm{MPa}$ for PVDF-20C and 


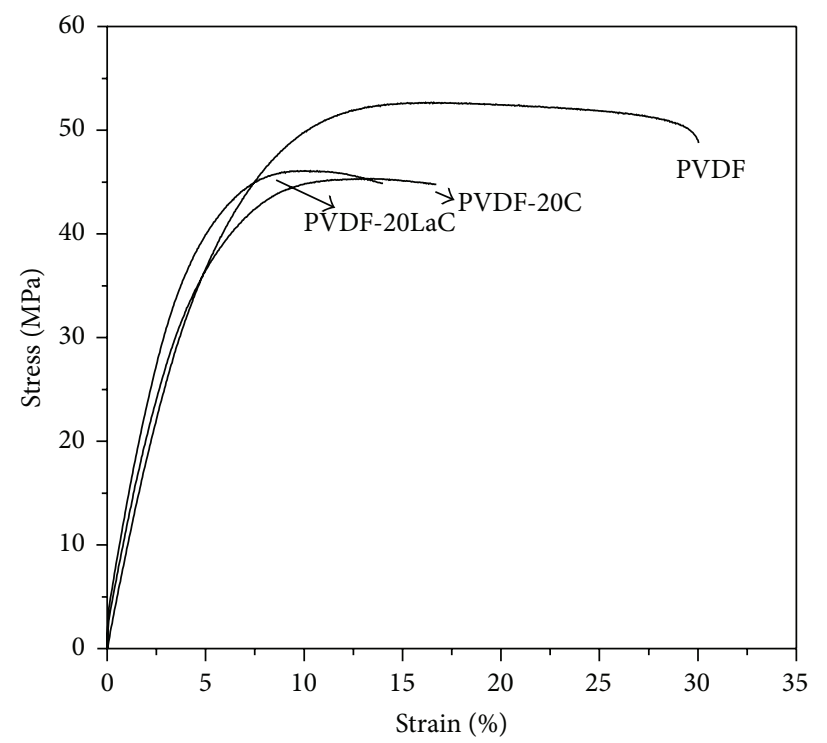

(a)

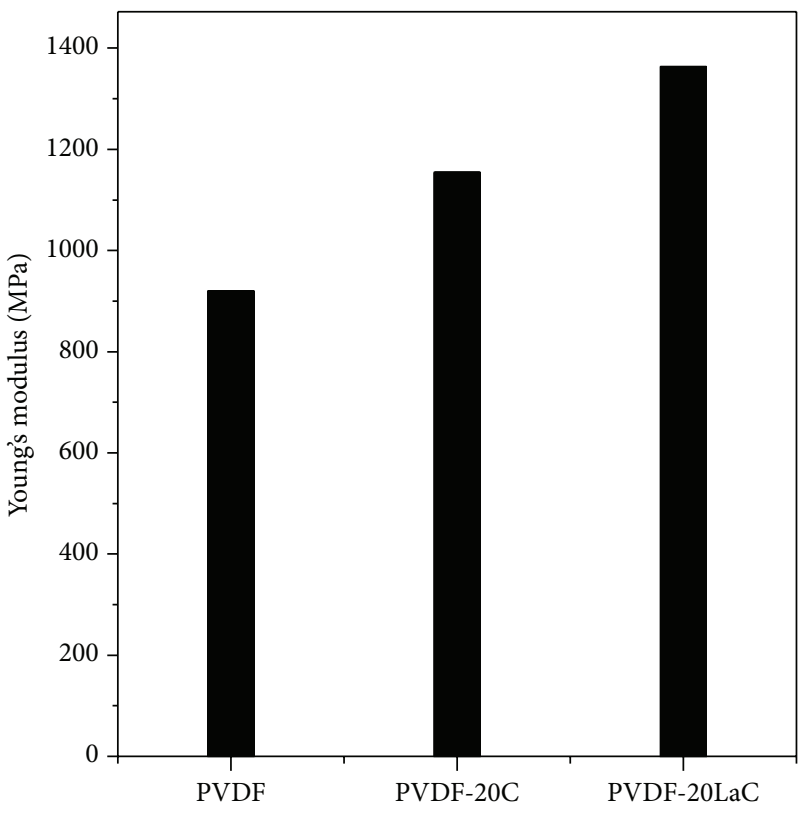

(b)

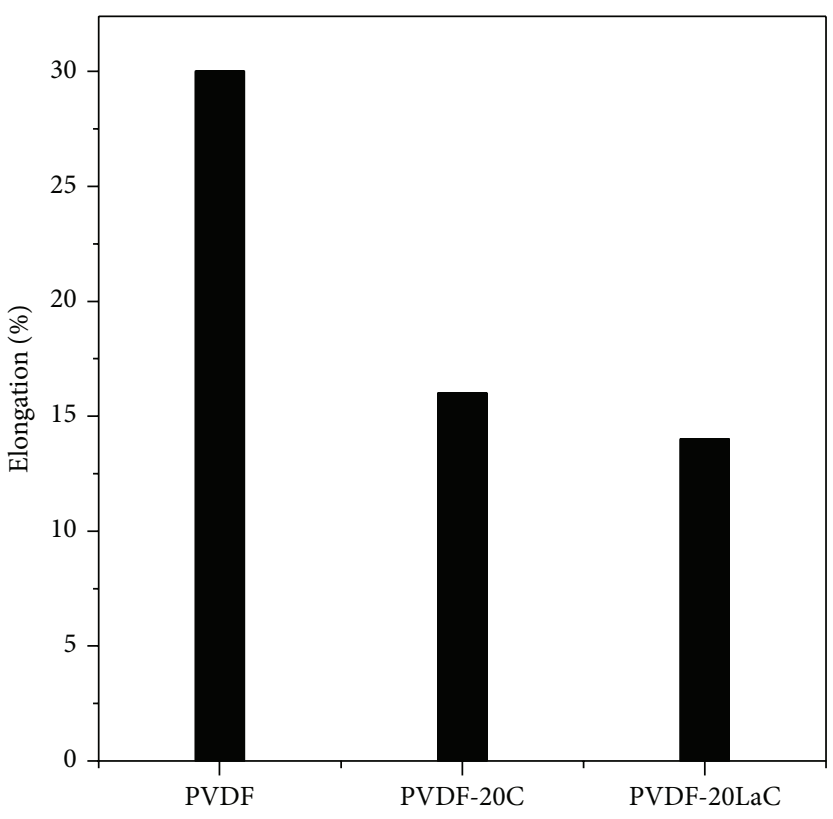

(c)

FIGURE 3: Stress-strain curves, Young's modulus, and elongation ((a)-(c)) for pure PVDF, PVDF-20C, and PVDF-20LaC composites.

PVDF-20LaC composites, respectively. This increment can be attributed to better interaction between stiffer ceramic and PVDF matrix thus making composites stiffer than pure PVDF. Elongation at breaking point decreased from $30 \%$ in case of PVDF to $16 \%$ and $14 \%$ in case of PVDF-20C and PVDF-20LaC composites, respectively. Ceramic particles act as defects from macroscopic point of view which inhibit the PVDF chains from packing with each other and result in the reduction in elongation at breaking point in the composites.

3.4. Dielectric Properties. Frequency dependence of dielectric constant of CCTO, LaCCTO, and composites is shown in Figures 4(a)-4(d). So many theories have been proposed to explain the abnormal dielectric properties of CCTO but the internal barrier layer capacitance mechanism has been widely accepted [15]. The intrinsic dielectric response in CCTO is due to barrier layer capacitance associated with grain boundaries which results in interfacial polarization. The giant permittivity depends on the microstructure and on formation of the internal boundary layer capacitance (IBLC) between the grains [25]. La doping in CCTO significantly modifies the grain boundaries leading to more efficient IBLC. Dielectric constant of CCTO is 3600 at $100 \mathrm{~Hz}$ and room temperature. This increases to 9900 in case of LaCCTO at $100 \mathrm{~Hz}$ and room temperature. It is noted that La doping in CCTO considerably increases the dielectric constant. 


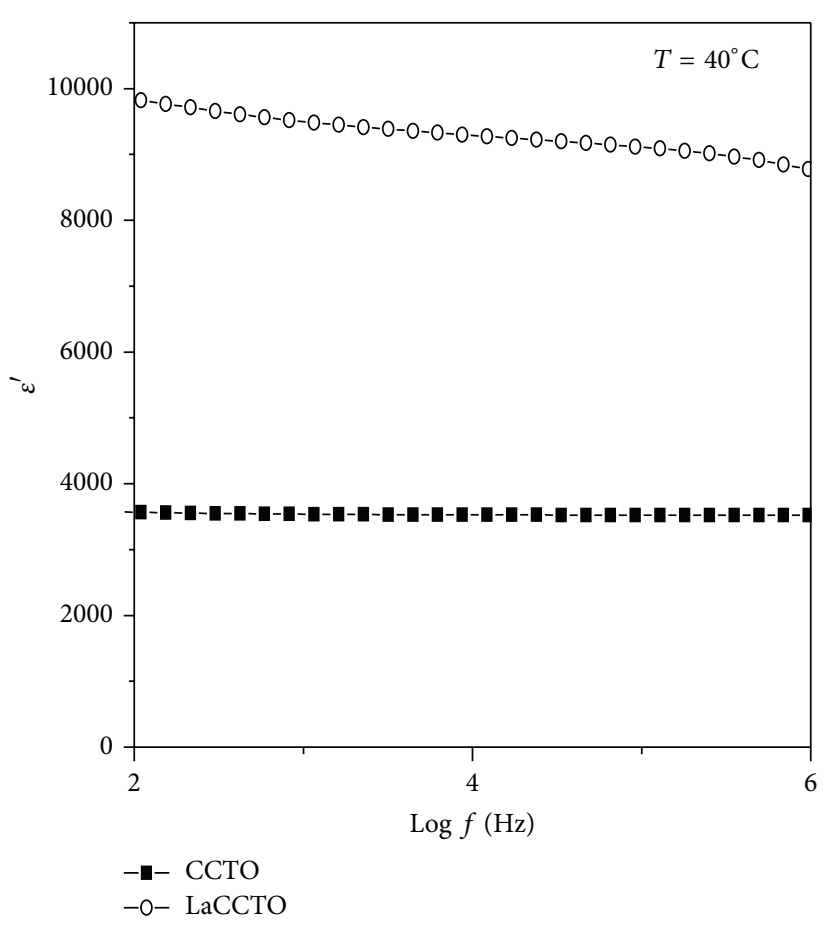

(a)

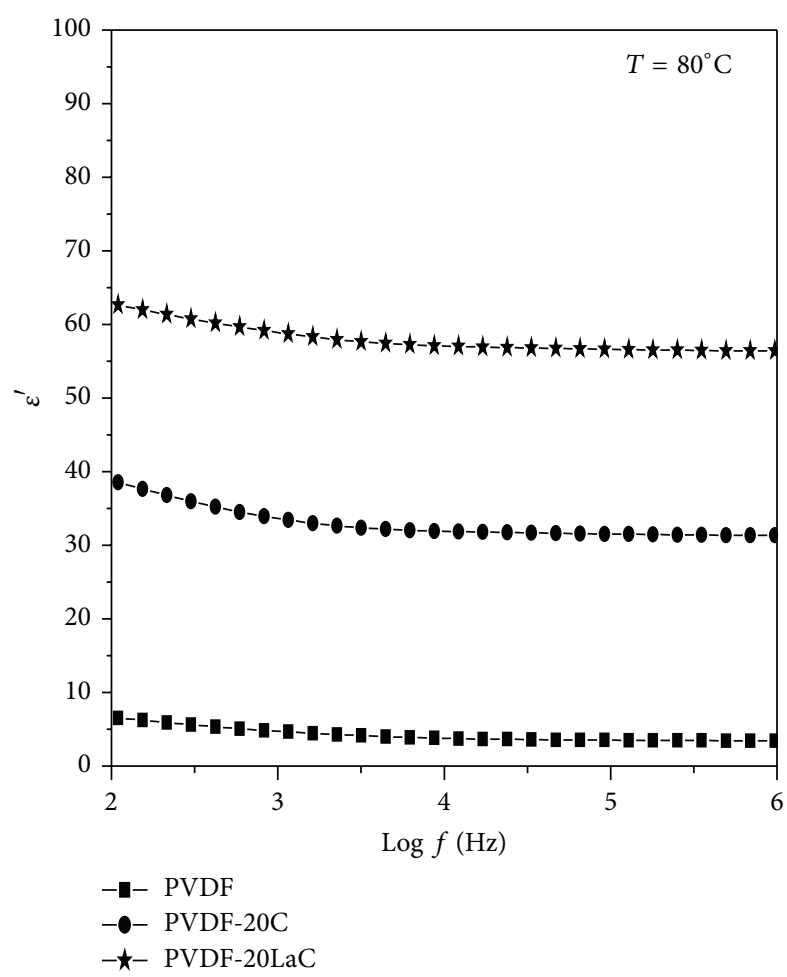

(c)

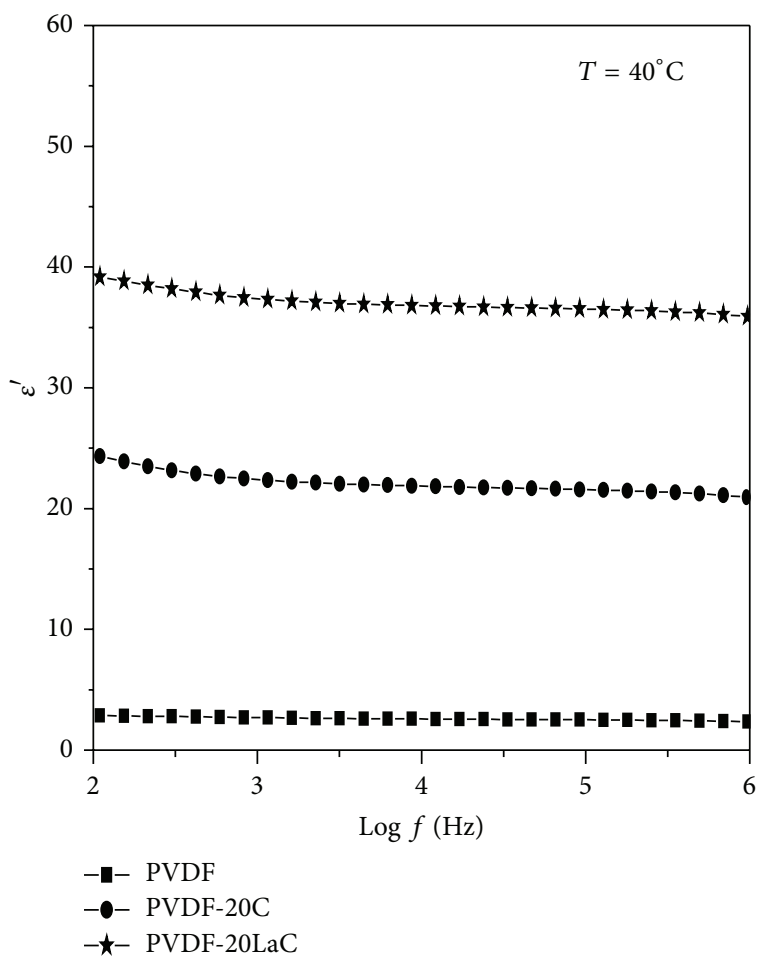

(b)

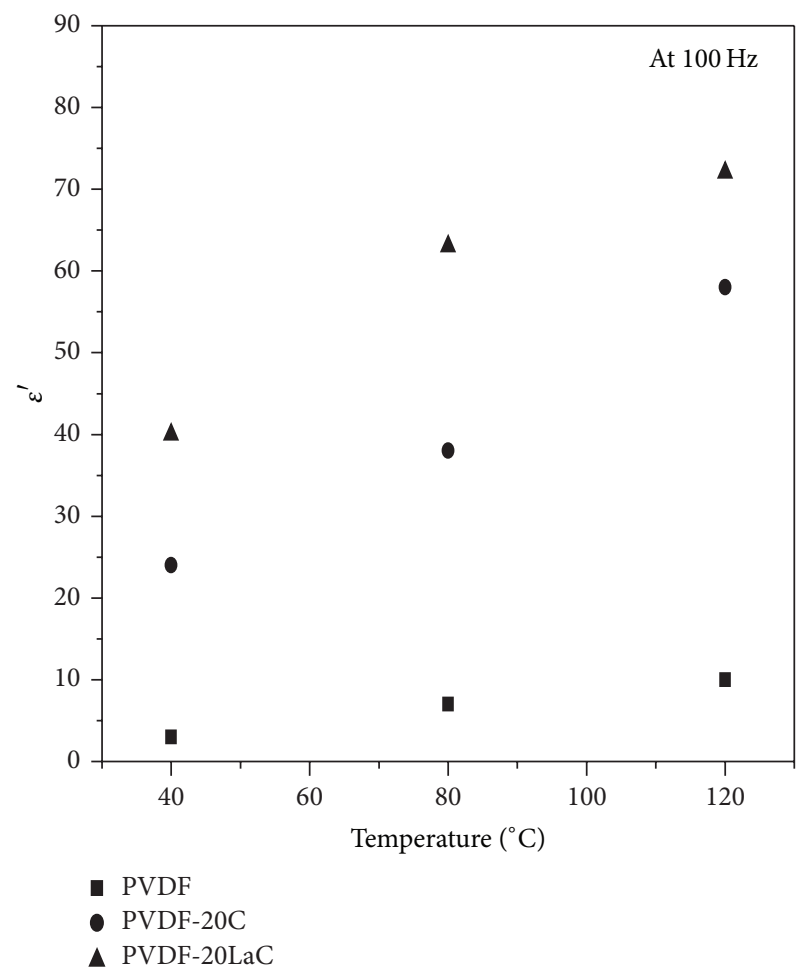

(d)

FIGURE 4: Frequency dependence of effective dielectric constant of CCTO and LaCCTO (a), PVDF, PVDF-20C, and PVDF-20LaC composites at $40^{\circ} \mathrm{C}(\mathrm{b})$, at $80^{\circ} \mathrm{C}(\mathrm{c})$, and at different temperature (d). 


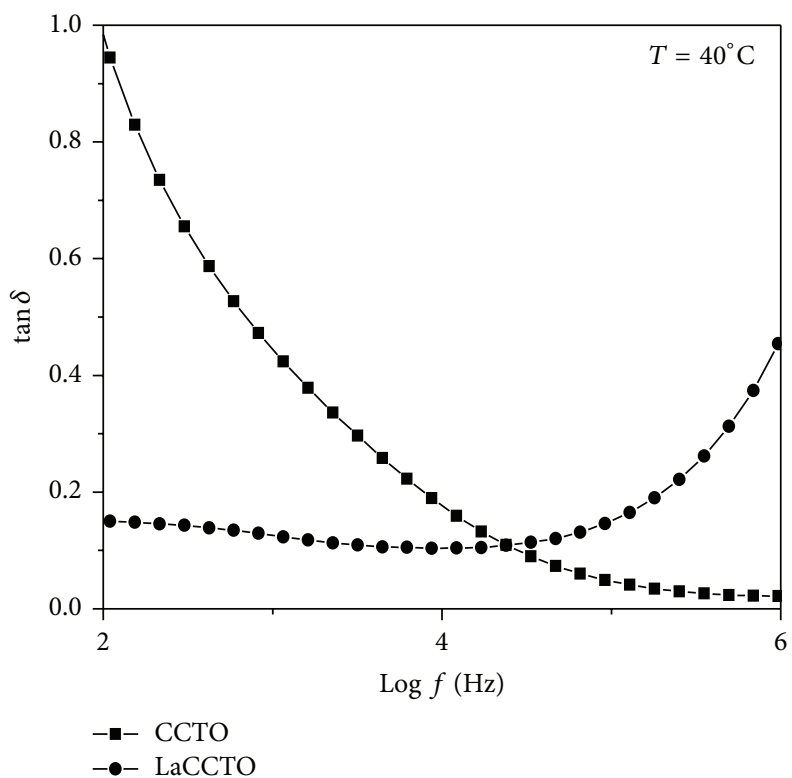

(a)

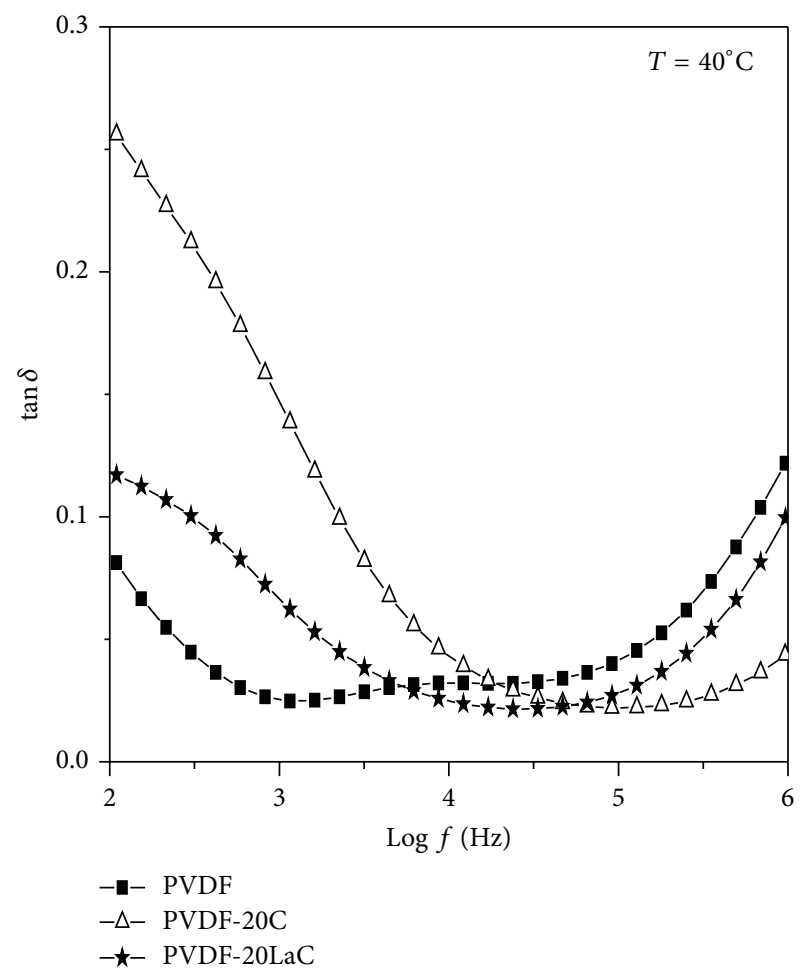

(b)

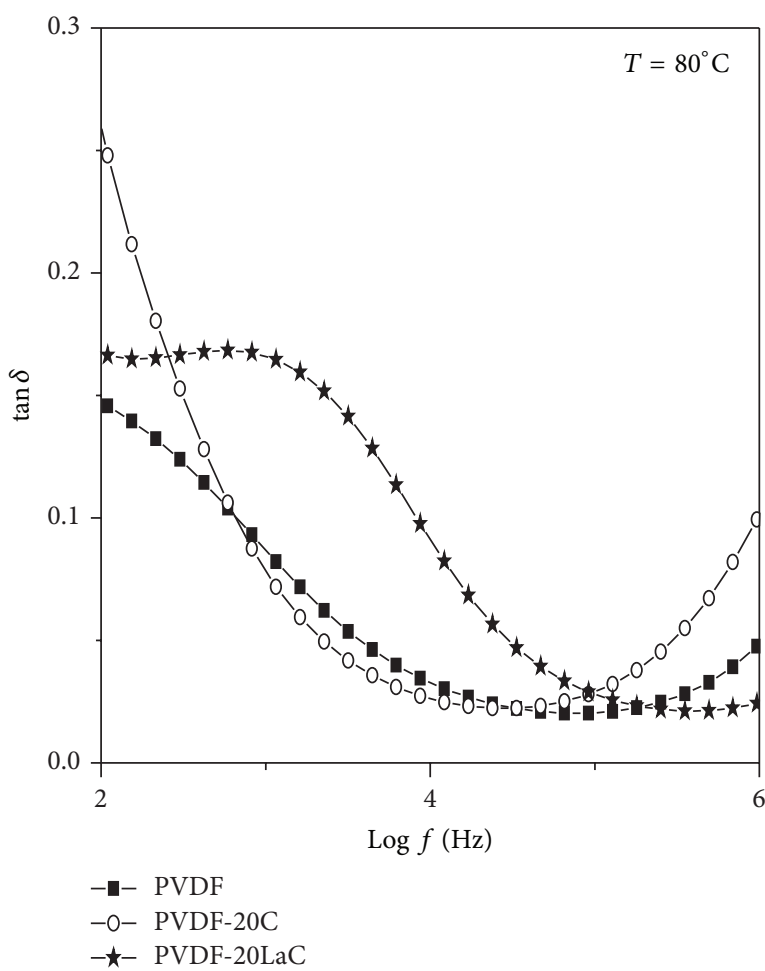

(c)

FIGURE 5: Frequency dependence of loss tangent of CCTO and LaCCTO (a), PVDF, PVDF-20C, and PVDF-20LaC composites at 40 ${ }^{\circ} \mathrm{C}$ (b) and $80^{\circ} \mathrm{C}(\mathrm{c})$.

Effective dielectric constant of PVDF increases with CCTO and LaCCTO dispersion. For PVDF-20C composite, dielectric constant increases to 24 from 3.5 of PVDF, whereas, in case of PVDF-20LaC composite, dielectric constant is 40 . In PVDF-20LaC composite increase in the dielectric constant is quite large. The dielectric constants of the ceramics as well as composites show weak frequency dependence in the range $\mathrm{I} \mathrm{KHz}-1 \mathrm{MHz}$. This is a desirable from the point of view of application in the devices. With increase in temperature there is an increase in dielectric constant. Increase in the value of $\varepsilon$ with temperature also supports this (Figure 4(d)). This shows that the difference between the conductivity of the polymer 


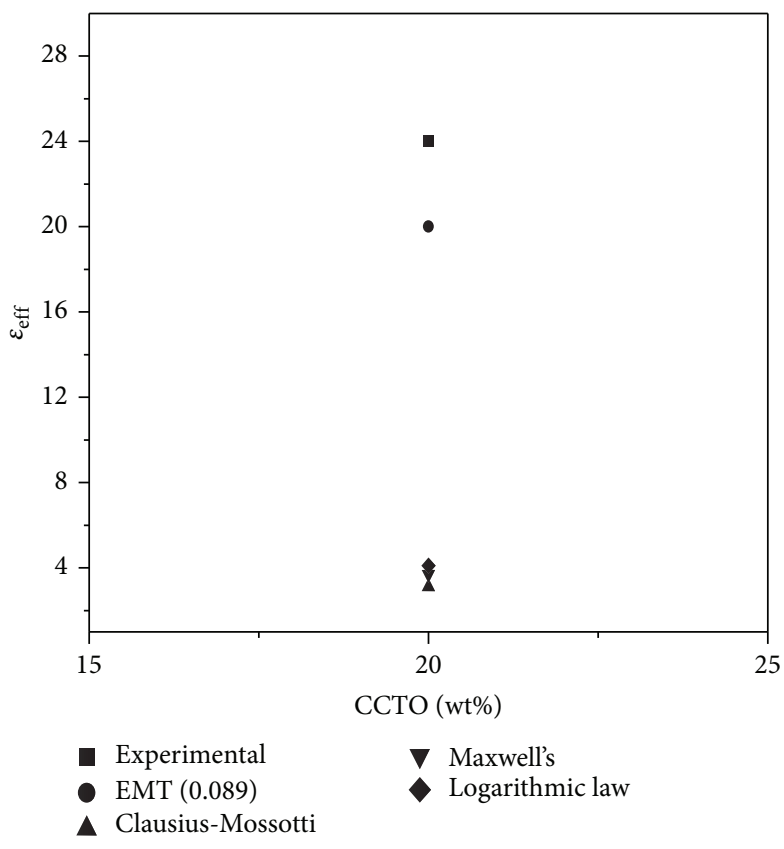

(a)

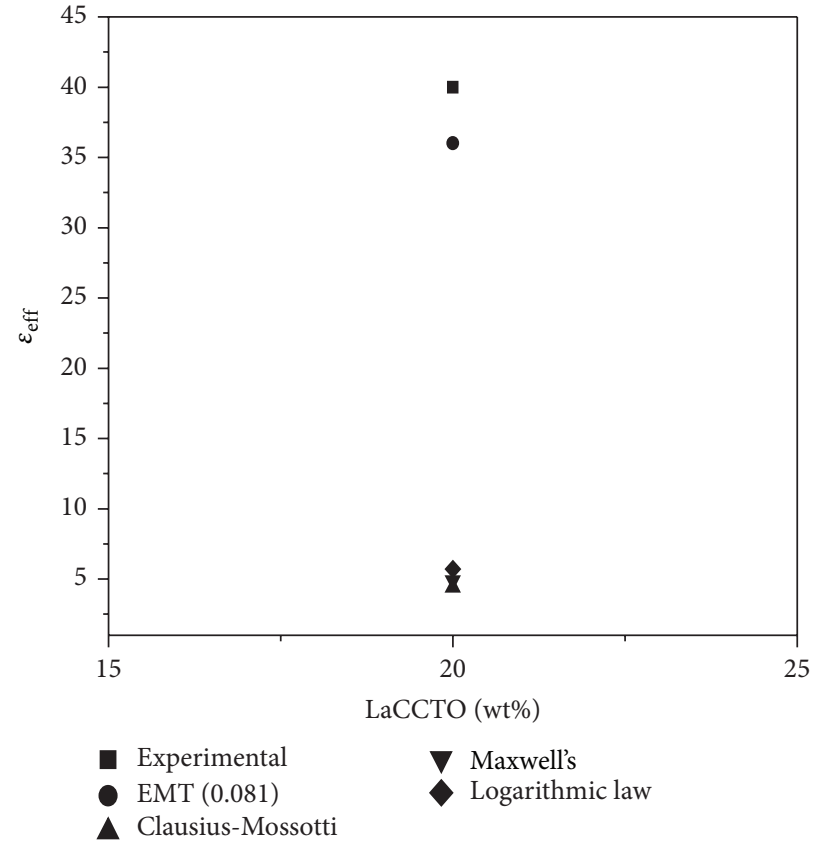

(b)

FIGURE 6: Variation of effective dielectric constant $\left(\varepsilon_{\text {eff }}\right)$ measured at $100 \mathrm{~Hz}$ and $40^{\circ} \mathrm{C}$ for PVDF-20C and PVDF-20LaC composites based on various models.

and the filler increases with rising temperature. The high $\varepsilon$ of the composites is mainly because of interfacial/space charge polarization and high dielectric constant of the ceramic fillers used. Increase in the dielectric constant of the polymer with ceramic dispersion can be explained as follows. Enhancement in dielectric constant of the composites is mainly because of the interfacial polarization and the high dielectric permittivity of CCTO and LaCCTO. In the polymers ceramic composites, interfacial polarization is always present due to difference in the conductivity of the polymer matrix and the ceramic fillers (CCTO and LaCCTO). Increase in the interfacial area with increasing filler content enhances the interfacial polarization and hence the dielectric permittivity.

Frequency dependence of dielectric loss $(\tan \delta)$ is shown in Figures 5(a)-5(c). Considerable decrease in the dielectric loss is observed with La doping in CCTO. Loss tangent value of CCTO and LaCCTO is 0.97 and 0.14 at $100 \mathrm{~Hz}$ and room temperature, respectively. A considerable decrease in the loss tangent is also observed in the composite. For PVDF, PVDF20C, and PVDF-20LaC, value of $\tan \delta$ is $0.08,0.25$, and 0.11 at $100 \mathrm{~Hz}$ at room temperature, respectively.

To predict the effective dielectric constant of the composites various models are used. The dielectric property of a diphasic dielectric mixture comprising spherical crystallites with high dielectric permittivity and a matrix of low dielectric permittivity can be described by Maxwell's model [32]. According to this model, the effective dielectric permittivity of the composite is given by

$$
\varepsilon_{\mathrm{eff}}=\frac{\delta_{p} \varepsilon_{p\left(2 / 3+\varepsilon_{c} / 3 \varepsilon_{p}\right)+\delta_{c} \varepsilon_{c}}}{\delta_{p}\left(2 / 3+\varepsilon_{c} / 3 \varepsilon_{p}\right)+\delta_{c}}
$$

where $\varepsilon_{c}, \varepsilon_{p}, \delta_{c}$, and $\delta_{p}$ are the dielectric constant of CCTO, LaCCTO, and PVDF and the volume fraction of the ceramic and the polymer, respectively. After substituting the values of $\varepsilon_{c}, \varepsilon_{p}, \delta_{c}$, and $\delta_{p}$, the values of $\varepsilon_{\text {eff }}$ obtained deviate much from the experimental values for the composites under study (Figure 6).

In the case of Clausius-Mossotti model [33], it is assumed that the mixture of dielectric is composed of spherical crystallites dispersed in a continuous medium. The effective dielectric constant $\left(\varepsilon_{\text {eff }}\right)$ of the composite is given by the following equation:

$$
\varepsilon_{\mathrm{eff}}=\varepsilon_{p}\left[1+3 \delta\left(\frac{\left(\varepsilon_{c}-\varepsilon_{p}\right)}{\varepsilon_{c}+2 \varepsilon_{p}}\right)\right] .
$$

Experimental values obtained deviate much from the predicted value of $\varepsilon_{\text {eff }}$ using this model (Figure 6). This may be due to nonspherical shape of ceramic particles as shown by SEM.

Lichtenecker's or logarithmic mixture rule is also used to predict the effective dielectric constant value [34]:

$$
\log \varepsilon_{\text {eff }}=\delta_{1} \log \varepsilon_{1}+\delta_{2} \log \varepsilon_{2} .
$$

Experimental results vary much from the predicted results using this model also (Figure 6).

The effective medium theory (EMT) model [35] has been developed taking into account the morphology of the particles. According to this model, the $\varepsilon_{\text {eff }}$ is given by

$$
\varepsilon_{\mathrm{eff}}=\varepsilon_{p}\left[1+\frac{f_{c}\left(\varepsilon_{c}-\varepsilon_{p}\right)}{\varepsilon_{p}+n\left(1-f_{c}\right)\left(\varepsilon_{c}-\varepsilon_{p}\right)}\right],
$$


where $f_{c}$ is the volume fraction of the ceramic dispersed and $\varepsilon_{c}, \varepsilon_{p}$, and $n$ are the dielectric constant of the ceramic, polymer, and the ceramic morphology fitting factor, respectively. The experimental values obtained are closest to the predicted values in this case of all the models employed to predict the $\varepsilon_{\text {eff }}$ values. The shape parameter $\mathrm{n}$ has been found to be 0.089 and 0.081 for PVDF-20C and PVDF-20LaC.

\section{Conclusion}

$\mathrm{CaCu}_{3} \mathrm{Ti}_{4} \mathrm{O}_{12}$ and $\mathrm{Ca}_{(1-3 x / 2)} \mathrm{La}_{x} \mathrm{Cu}_{3} \mathrm{Ti}_{4} \mathrm{O}_{12}(x=0.05)$ were successfully prepared by semiwet route. Extrusion process was used to fabricate $20 \mathrm{wt} \% \mathrm{CCTO}$ and $\mathrm{LaCCTO} / \mathrm{PVDF}$ (PVDF-20C and PVDF-20LaC) composites. XRD patterns indicate that there is no secondary phase present in CCTO and LaCCTO. Morphological studies reveal the homogeneous distribution of CCTO and LaCCTO fillers in PVDF matrix. Young's modulus in the composites is significantly higher than that of the polymer. With addition of CCTO, dielectric constant of matrix PVDF increases substantially. This increase in dielectric constant is more in the case of LaCCTO addition as compared to that of CCTO addition. Loss tangent is less in the case of PVDF-20LaC composites in comparison to PVDF-20C composites. The results obtained indicate that La doping on Ca site proved effective in increasing the dielectric permittivity and decreasing the dielectric loss. The PVDF-20LaC composite exhibits better dielectric and mechanical properties. So it can be concluded that PVDF dispersed with La doped CCTO can be a potential material for developing embedded capacitors for microelectronics applications.

\section{Conflict of Interests}

The authors declare that there is no conflict of interests regarding the publication of this paper.

\section{References}

[1] R. Popielarz, C. K. Chiang, R. Nozaki, and J. Obrzut, "Dielectric properties of polymer/ferroelectric ceramic composites from $100 \mathrm{~Hz}$ to $10 \mathrm{GHz}$," Macromolecules, vol. 34, no. 17, pp. 5910-5915, 2001.

[2] N. Korotkov, S. A. Gridnev, S. A. Konstantinov, T. I. Klimentova, Y. V. Barmin, and I. V. Babkina, "Dielectric permittivity and conductivity of amorphous $\mathrm{PbTiO}_{3}$," Ferroelectrics, vol. 299, pp. 171-177, 2004.

[3] L. Jylhä, J. Honkamo, H. Jantunen, and A. Sihvola, "Microstructure-based numerical modeling method for effective permittivity of ceramic/polymer composites," Journal of Applied Physics, vol. 97, no. 10, Article ID 104104, 2005.

[4] Z.-M. Dang, J.-B. Wu, L.-Z. Fan, and C.-W. Nan, "Dielectric behavior of Li and Ti co-doped NiO/PVDF composites," Chemical Physics Letters, vol. 376, no. 3-4, pp. 389-394, 2003.

[5] Y. K. Sung and F. El-Tantawy, "Novel smart polymeric composites for thermistors and electromagnetic wave shielding effectiveness from TiC loaded styrene-butadiene rubber," Macromolecular Research, vol. 10, no. 6, pp. 345-358, 2002.
[6] L. Ramajo, M. S. Castro, and M. M. Reboredo, "Effect of silane as coupling agent on the dielectric properties of $\mathrm{BaTiO}_{3}$ epoxy composites," Composites Part A: Applied Science and Manufacturing, vol. 38, no. 8, pp. 1852-1859, 2007.

[7] Z.-M. Dang, Y.-F. Yu, H.-P. Xu, and J. Bai, "Study on microstructure and dielectric property of the $\mathrm{BaTiO}_{3}$ /epoxy resin composites," Composites Science and Technology, vol. 68, no. 1, pp. 171$177,2008$.

[8] C. V. Chanmal and J. P. Jog, "Dielectric relaxations in PVDF/ $\mathrm{BaTiO}_{3}$ nanocomposites," Express Polymer Letters, vol. 2, no. 4, pp. 294-301, 2008.

[9] Q. Li, Q. Z. Xue, X. L. Gao, and Q. B. Zheng, "Temperature dependence of the electrical properties of the carbon nanotube/polymer composites," Express Polymer Letters, vol. 3, no. 12, pp. 769-777, 2009.

[10] L. A. Ramajo, A. A. Cristóbal, P. M. Botta, J. M. Porto López, M. M. Reboredo, and M. S. Castro, "Dielectric and magnetic response of $\mathrm{Fe}_{3} \mathrm{O}_{4}$ /epoxy composites," Composites Part A: Applied Science and Manufacturing, vol. 40, no. 4, pp. 388-393, 2009.

[11] A. Soulintzis, C. Kontos, P. Karahaliou, C. C. Psarras, S. N. Ceorga, and C. A. Krontiras, "Dielectric relaxation processes in epoxy resim-ZnO composites," Journal of Polymer Science Part B: Polymer Physics, vol. 47, no. 4, pp. 445-454, 2009.

[12] C. G. Raptis, A. Patsidis, and G. C. Psarras, "Electrical response and functionality of polymer matrix-titanium carbide composites," Express Polymer Letters, vol. 4, no. 4, pp. 234-243, 2010.

[13] Y. Roichman, M. S. Silverstein, A. Siegmann, and M. Narkis, "Percolation of electrical conductivity in solution-cast blends containing polyaniline," Journal of Macromolecular Science: Physics, vol. 38, no. 1-2, pp. 145-161, 1999.

[14] K. Mazur, Ferroelectric Polymers: Chemistry, Physics and Applications, Marcel Dekker, New York, NY, USA, 1995.

[15] Y. Yan, L. Jin, L. Feng, and G. Cao, "Decrease of dielectric loss in giant dielectric constant $\mathrm{CaCu}_{3} \mathrm{Ti}_{4} \mathrm{O}_{12}$ ceramics by adding $\mathrm{CaTiO}_{3}$," Materials Science and Engineering B: SolidState Materials for Advanced Technology, vol. 130, no. 1-3, pp. 146-150, 2006.

[16] J. Kułek, I. Szafraniak, B. Hilczer, and M. Połomska, "Dielectric and pyroelectric response of PVDF loaded with $\mathrm{BaTiO}_{3}$ obtained by mechanosynthesis," Journal of Non-Crystalline Solids, vol. 353, no. 47-51, pp. 4448-4452, 2007.

[17] Y. Bai, Z.-Y. Cheng, V. Bharti, H. S. Xu, and Q. M. Zhang, "High-dielectric-constant ceramic-powder polymer composites," Applied Physics Letters, vol. 76, no. 25, pp. 3804-3806, 2000.

[18] A. M. Glazer, "The classification of tilted octahedra in perovskites," Acta Crystallographica Section B: Structural Crystallography and Crystal Chemistry, vol. 28, no. 11, pp. 3384-3392, 1972.

[19] A. P. Ramirez, M. A. Subramanian, M. Gardel et al., "Giant dielectric constant response in a copper-titanate," Solid State Communications, vol. 115, no. 5, pp. 217-220, 2000.

[20] Y. L. Zhao, G. W. Pan, Q. B. Ren, Y. G. Cao, L. X. Feng, and Z. K. Jiao, "High dielectric constant in $\mathrm{CaCu}_{3} \mathrm{Ti}_{4} \mathrm{O}_{12}$ thin film prepared by pulsed laser deposition," Thin Solid Films, vol. 445, no. 1, pp. 7-13, 2003.

[21] S.-Y. Chung, I.-D. Kim, and S.-J. L. Kang, "Strong nonlinear current-voltage behaviour in perovskite-derivative calcium copper titanate.," Nature materials, vol. 3, no. 11, pp. 774-778, 2004. 
[22] M. Arbatti, X. Shan, and Z. Cheng, "Ceramic-polymer composites with high dielectric constant," Advanced Materials, vol. 19, no. 10, pp. 1369-1372, 2007.

[23] C. Yang, H. S. Song, and D. B. Liu, "Effect of coupling agents on the dielectric properties of $\mathrm{CaCu}_{3} \mathrm{Ti}_{4} \mathrm{O}_{12}$ /PVDF composites," Composites Part B: Engineering, vol. 50, pp. 180-186, 2013.

[24] L. Zhang, X. Shan, P. Wu, and Z. Y. Cheng, "Dielectric characteristics of $\mathrm{CaCu}_{3} \mathrm{Ti}_{4} \mathrm{O}_{12} / \mathrm{P}$ (VDF-TrFE) nanocomposites," Applied Physics A: Materials Science and Processing, vol. 107, no. 3, pp. 597-602, 2012.

[25] L. A. Ramajo, M. A. Ramírez, P. R. Bueno, M. M. Reboredo, and M. S. Castro, "Dielectric behaviour of $\mathrm{CaCu}_{3} \mathrm{Ti}_{4} \mathrm{O}_{12}$ - Epoxy composites," Materials Research, vol. 11, no. 1, pp. 85-88, 2008.

[26] P. Thomas, K. T. Varughese, K. Dwarakanath, and K. B. R. Varma, "Dielectric properties of Poly(vinylidene fluoride)/ $\mathrm{CaCu}_{3} \mathrm{Ti}_{4} \mathrm{O}_{12}$ composites," Composites Science and Technology, vol. 70, no. 3, pp. 539-545, 2010.

[27] W. Yang, S. Yu, R. Sun, and R. Du, "Nano- and microsize effect of CCTO fillers on the dielectric behavior of CCTO/PVDF composites," Acta Materialia, vol. 59, no. 14, pp. 5593-5602, 2011.

[28] Z.-M. Dang, T. Zhou, S.-H. Yao et al., "Advanced calcium copper titanate/polyimide functional hybrid films with high dielectric permittivity," Advanced Materials, vol. 21, no. 20, pp. 2077-2082, 2009.

[29] B. S. Prakash and K. B. R. Varma, "Dielectric behavior of CCTO/epoxy and Al-CCTO/epoxy composites," Composites Science and Technology, vol. 67, no. 11-12, pp. 2363-2368, 2007.

[30] A. Srivastava, P. Maiti, D. Kumar, and O. Parkash, "Mechanical and dielectric properties of $\mathrm{CaCu}_{3} \mathrm{Ti}_{4} \mathrm{O}_{12}$ and $\mathrm{La}$ doped $\mathrm{CaCu}_{3} \mathrm{Ti}_{4} \mathrm{O}_{12}$ poly(vinylidene fluoride) composites," Composites Science and Technology, vol. 93, pp. 83-89, 2014.

[31] A. Srivastava, K. Kumar Jana, P. Maiti, D. Kumar, and O. Parkash, "Mechanical and dielectric behaviour of $\mathrm{CaCu}_{3} \mathrm{Ti}_{4} \mathrm{O}_{12}$ and $\mathrm{Nb}$ doped $\mathrm{CaCu}_{3} \mathrm{Ti}_{4} \mathrm{O}_{12}$ poly(vinylidene fluoride) composites," Journal of Composites, vol. 2014, Article ID 769379, 9 pages, 2014.

[32] J. C. Maxwell, A Treatise on Electricity and Magnetism, Dover, New York, NY, USA, 1954.

[33] H. Frolich, Theory of Dielectrics, Clarendon Press, Oxford, UK, 1949.

[34] H. S. Nalwa, Ferroelectric Polymers, Chapter 11, Marcel Dekker, New York, NY, USA, 1995.

[35] Y. Rao, J. Qu, T. Marinis, and C. P. Wong, "A precise numerical prediction of effective dielectric constant for polymer-ceramic composite based on effective-medium theory," IEEE Transactions on Components and Packaging Technologies, vol. 23, no. 4, pp. $680-683,2000$. 

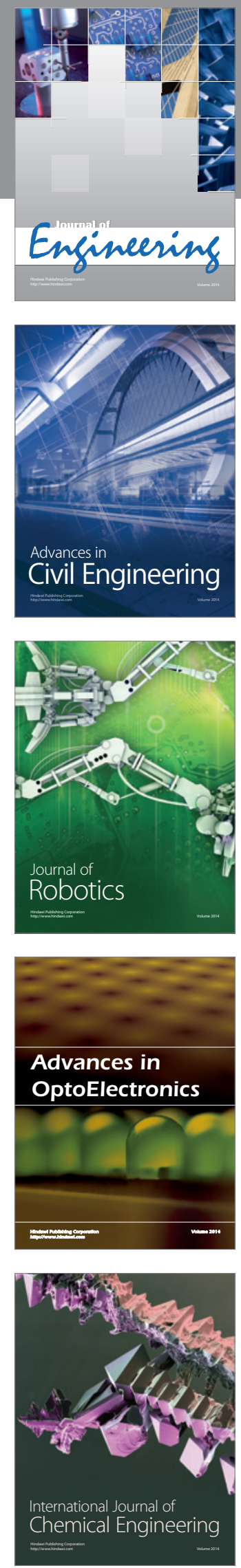

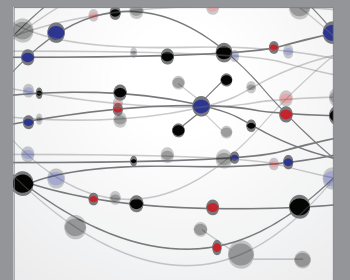

The Scientific World Journal
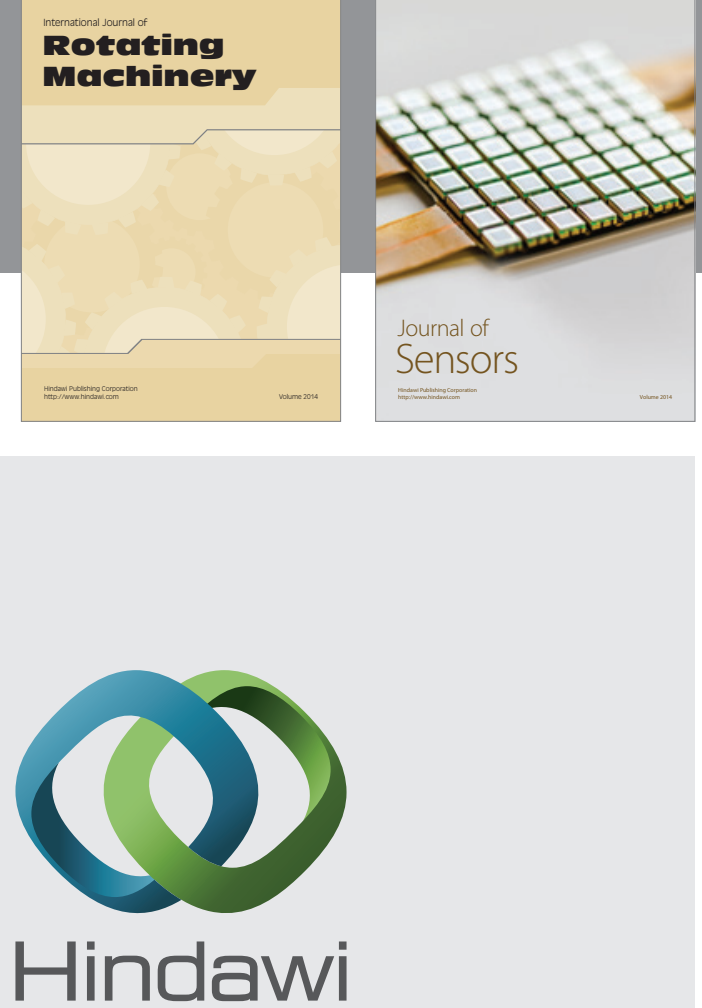

Submit your manuscripts at http://www.hindawi.com
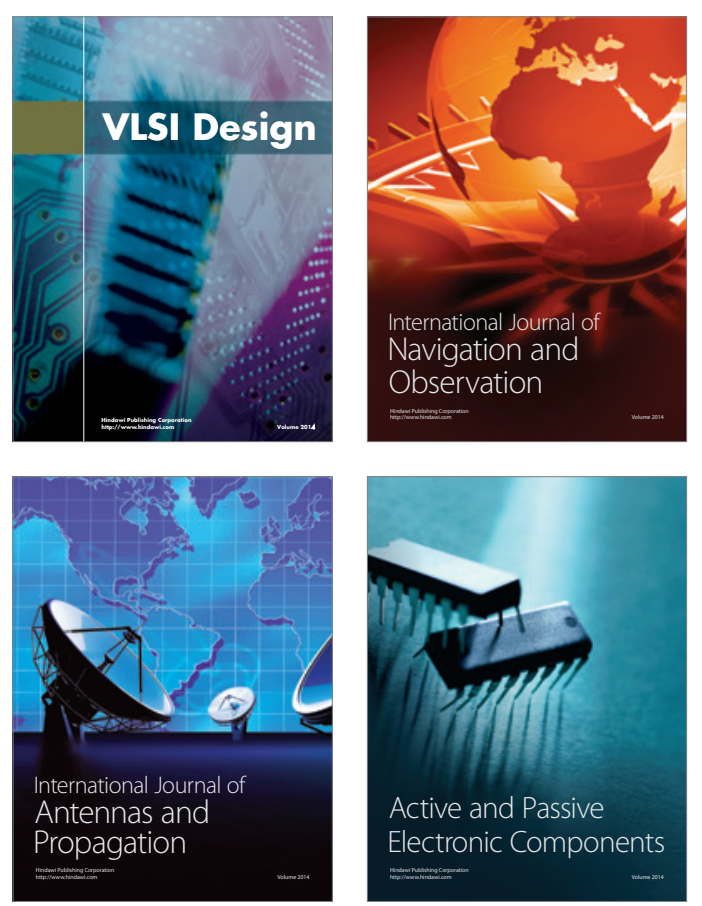
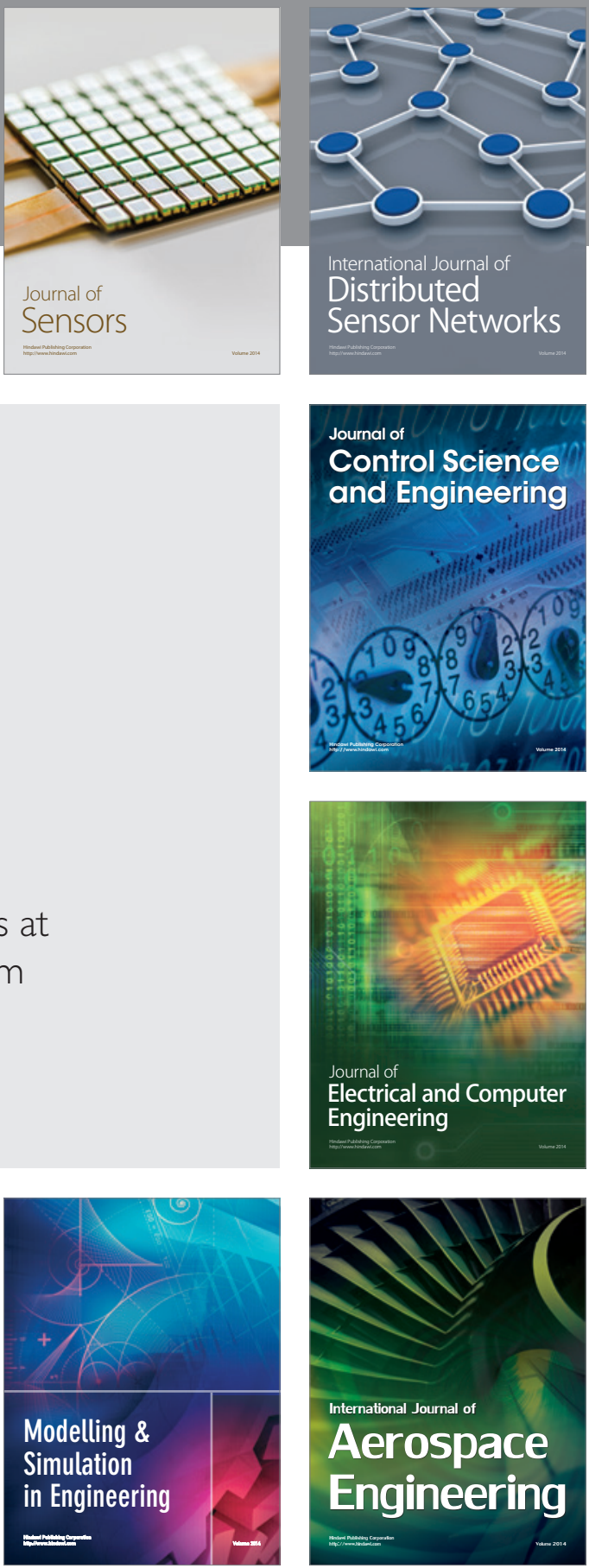

Journal of

Control Science

and Engineering
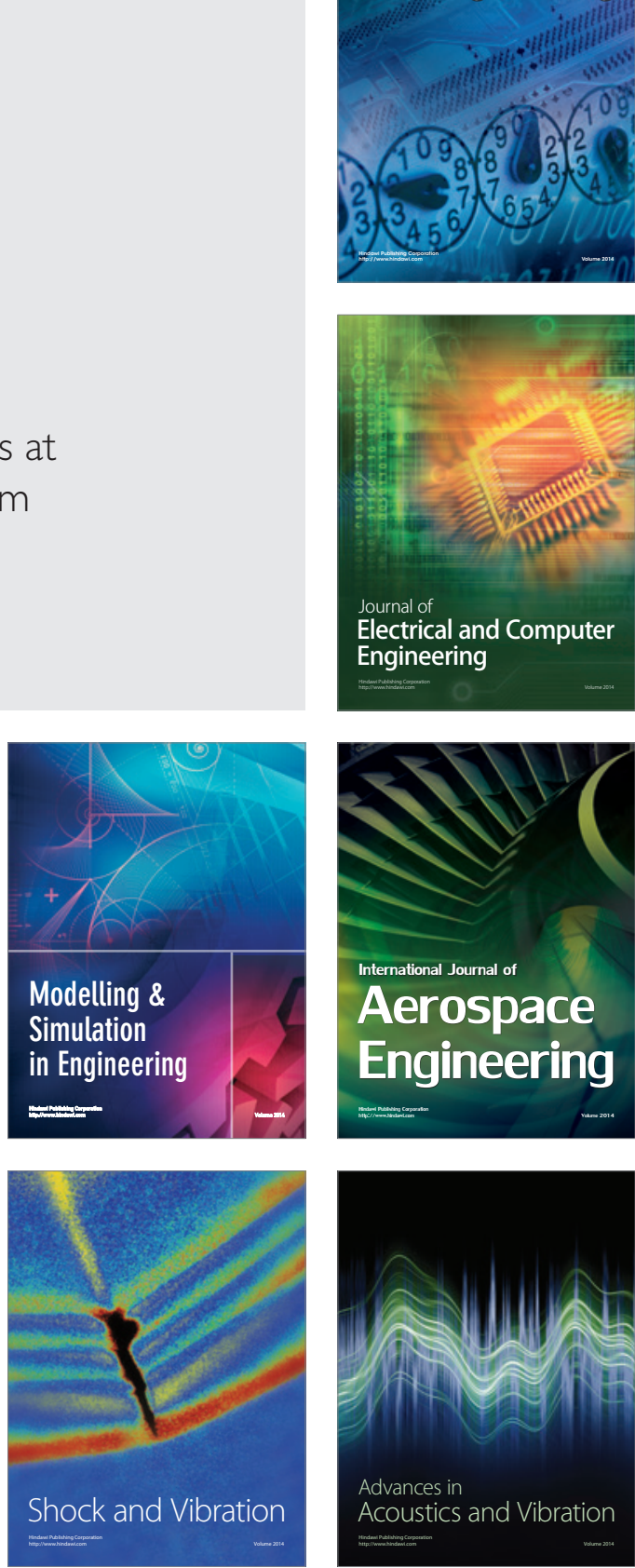